\title{
Hypoxia predicts relapse and recurrence after radiotherapy
}

Tumor hypoxia is associated with early biochemical relapse after radiotherapy for prostate cancer and with local recurrence, according to a new study from a team in Toronto, Canada.

Studies have suggested that hypoxia modulates tumor behavior and treatment response, promoting both aggressiveness and metastasis. "Many kinds of tumors are hypoxic, but it has never conclusively been demonstrated before in prostate cancer," comments Michael Milosevic, the corresponding author on the study.

\section{4 ...patients with more-hypoxic} tumors were more likely to relapse... 77

247 men were included in the series, all of whom had clinically localized prostate cancer. Hypoxia (hypoxic percentage below $10 \mathrm{mmHg}\left[\mathrm{HP}_{10}\right]$ )was measured before the men underwent radiotherapy, using an ultrasonography-guided transrectal needle-electrode technique. Patients were followed up at 6-monthly intervals with clinical examination and serum PSA measurement.

Biochemical failure (using the Phoenix definition of a $2 \mathrm{ng} / \mathrm{ml}$ increase in serum PSA above the nadir) was used as the primary end point, with local recurrence as a secondary end point. Biochemical failure was identified in 79 patients, with a corresponding biochemical relapse-free rate (bRFR) of $78 \%$ at 5 years.

Gleason score and PSA level were used to determine a baseline clinical model and $\mathrm{HP}_{10}$ then added to the model to determine its independent predictive value. Univariate analysis showed $\mathrm{HP}_{10}$ to have a maximal effect on bRFR at earlier time points, which diminished with increasing follow-up duration, suggesting that patients with more-hypoxic tumors were more likely to relapse in the first 48 months after treatment, an effect that remained significant even after correcting for Gleason score and PSA.

70 patients also underwent follow-up biopsies to determine local tumor control. 36 of these patients had biochemical failure, 31 of whom had a local recurrence of prostate cancer on follow-up biopsy. Hypoxia was the only factor predictive of local recurrence in these patients, an effect that was, once again, strongest in early follow-up.

The team hope that a greater understanding of hypoxia and prostate cancer behavior might lead to the development of new treatment strategies. "We hope to explore this concept of new drugs that might target hypoxia or manifestations of hypoxia so treatments are more effective," says Milosevic.

\section{Annette Fenner}

Original article Milosevic, M. et al. Prostate hypoxia and biochemical failure. Clin. Cancer Res. 18, 1-7 (2012) 